\title{
Looking Forward, Respecting the Past
}

\author{
Brett Paull ${ }^{1}$
}

Published online: 13 June 2017

(C) Springer-Verlag GmbH Germany 2017

It is with a feeling of considerable excitement that I begin a new role as a Scientific Editor for Chromatographia. It may surprise some readers, but Chromatographia is rapidly approaching its 50th birthday, with its first issue published in 1968, close to a very special date in my own calendar, as I arrived on the scene 12 months later. The first Editorial piece published within that first issue was penned by $\mathrm{D}$. R. Deans, L. Szepesy, G. Guiochon, and R. Kaiser, each of which were pioneers at the time, and some of which grew into giants of separation science. Back in its early days, Chromatographia also published papers in any of three languages, English, German, and French, and the Editors were very keen to set the journal as a vehicle for rapid information exchange, noting competitive journals at that time had publication delays of 6-12 months, and that symposiums were, in their words, "sometimes too highly organised, so that-quite unintentionally-the scope of the discussions is restricted by time, and subject matter, quite apart from psychological factors. Accordingly, the yield of information is poor".

Since this time, a great deal has changed, and a great deal remains the same. Technology, in general, has advanced beyond recognition, yet the basic principles and some might say, basic equipment of both gas and liquid chromatography remains pretty much as it was. In his 1969 Editorial entitled 'Why buy a computer to operate a gas chromatographs?', Editor D. R. Deans clearly had an eye on the future. Amusingly, Editor Deans highlighted in

Brett Paull

brett.paull@utas.edu.au

1 Australian Centre for Research on Separation Sciences (ACROSS), School of Physical Sciences, University of Tasmania, Private Bag 75, Hobart, TAS 7001, Australia his discussion piece an issue that remains to this day, noting that "The typical (computer) panel .... is quite unsatisfactory .... as it incorporates a large number of separate controls and illuminated signs. The calibre of the person needed to operate these panels is higher than that often required to operate the gas chromatograph...". Any modern GC or HPLC user today confronted with the typical complexity of the modern operating system may well agree with these early sentiments.

So yes, I believe that it is very fair to say that certain aspects of separation technology have developed faster than others. Clearly, a few users today would still prefer a chart recorder to the PC-based system control. However, the number of still active HPLC systems around the world using pumps based upon the 1970s and 80s technology numbers in the 10s if not 100s of thousands, because, of course, they do the job and do the job well. So what can we predict today for the future of separation science? Well, for many years, now, the separation science community has pondered upon three future challenges, which we can loosely cluster under the themes of speed, portability, and ease of use. Of course, all developments are ultimately application driven, as if there is no immediate need, technology progresses slowly, or new technology becomes a 'flash in the pan' and quickly fades away. In the area of gas chromatography, the need for portable robust instrumentation for application in industrial settings has driven that technology forward along those thematic lines. 'Sample in - answer out' is the ever louder call from industry, with demand for instant information overtaking the traditional 'send the sample to the lab' mentality. However, in liquid chromatography, we have not seen as much progression, although of late, other issues, such as the cost of solvents, and the green chemistry movement, have seen the desire to rid separation laboratories of the typical endless rows 
of half-full solvent reservoirs. The background technology to deliver a hand-held HPLC already exists, and when flagged as a possibility to the traditional industry users, the item is met with 'I can think of a good application for that straight away!'. The ever powerful smartphone platform is beginning to provide greater analytical and diagnostic capabilities through add-ons, and who knows where this will eventually lead. Having said this, the law of inertia is incontestable, and to quote Mark Twain, "The less there is to justify a traditional custom, the harder it is to get rid of it". Major users of separation technology, such as the massive pharmaceutical industries, are often reluctant to jump first with any new development, and analogies of ocean super-tankers attempting to change course come to mind. However, we must have faith in the research and development to overcome such inertia, and so in writing this today, I look forward with enthusiasm to reading an Editorial 10 years from now which summarises the great advances made and significant societal problems solved by the separation science community.

So what does the future hold for Chromatographia? The weight of its history cements Chromatographia's place amongst the separation science-based journals for the foreseeable future. Going forward, we will be striving to place Chromatographia front and centre in the minds of all prospective authors, particularly young and emerging leaders and innovators in the field. As per the original 1968 Editorial, we will continue to provide authors with a rapid and excellent publishing experience, and a range of contribution formats, namely original articles, short communications, reviews, and letters to the Editor. However, I believe that a journal's role is more than just providing the published article. It should be a forum for discussion, new ideas, a diverse supporting platform for the scientific community. Like most journals, going forward, we will inevitably see increased electronic content, perhaps, more interactive content, and more engaging and exciting content. We will strive to be more receptive to ideas and needs of authors, and, in turn, provide content which will inspire the readership, steer them in new directions, provide the clue to solving their problems, and educate them on the current status quo.

So, it is with great excitement that I sign off on this first Editorial for Chromatographia, and look forward corresponding and meeting with many of our expert authors' in the future years. 